\title{
THE ASSOCIATION BETWEEN EXPRESSION OF KI-67 AND MMP-7 PROTEINS AND LYMPH NODE METASTASIS IN MALIGNANT SALIVARY GLAND TUMORS
}

\author{
Enas Alaa El-Din Abd El-Aziz*
}

\begin{abstract}
Aim of the work: To analyze the expression of both Ki-67 and MMP-7 proteins in malignant salivary gland tumors by immunohistochemistery and correlate the results with lymph node metastasis and some clinical parameters including sex, site and type of the tumor.
\end{abstract}

Material and Methods: The study was retrospectively designed. Thirty formalin fixed paraffin embedded blocks of different malignant salivary gland tumors were stained immunohistochemically for both Ki-67 and MMP-7. Immunostainig was evaluated and results were analyzed statistically in relation to lymph node metastasis and some clinical parameters as sex, site and type of the tumor. $P$ Value $\leq 0.05$ was considered significant.

Results: High Ki-67 ( $\mathrm{P}=0.0001)$ and MMP-7 expression $(\mathrm{P}=0.0001)$ was associated with lymph node metastasis. Ki-67 and MMP-7 immunostaining was not affected by either sex or site of the tumor but there was high association with type of the tumor.

Conclusion: MMPs and Ki-67 may have prognostic impact in malignant salivary gland tumors and can be used in molecular targeted therapy.

KEYWORDS : Ki-67, MMP-7, Salivary gland tumors.

\section{INTRODUCTION}

Malignant Salivary gland tumors (MSGTs) in head and neck account for nearly $2 \%$ to $4 \%$ of all oral cancers (incidence of 1-2 per100,000). MSGTs are more prevalent in major salivary glands, particularly the parotid (20\%-25\%) (A.L.D. Kruse et al, 2010).

Mucoepidermpoid carcinoma is the most common type of salivary gland cancer representing about $25-35 \%$. Other types include adenoid cystic carcinoma (ACC), acinic cell carcinoma, carcinoma ex-pleomorphic adenoma, polymorphous low grade adenocarcinoma (PLGA) and some newly discovered salivary gland tumors (P.C.Anila, 2014).

Risk factors for MSGTs include cigarette smoking, viral infections, rubber manufacturing workers and genes while ionizing radiation is among the well-established risk factors for MSGTs (A. Rousseau and C. Badoual, 2011).

\footnotetext{
* Lecturer of Oral and Maxillofacial Pathology, Faculty of Dentistry, Minia University.
} 
Malignant salivary gland tumors are rare and show complex histopathological pattern. Thus, there is a problem in predicting their clinical course by means of their recurrence, metastasis and survival rate.

It's important to predict the proliferating activities in tumors to predict their biological behavior (A. Abdel Aziz and M.M. Amin, 2012). Ki-67, one of proliferating markers, has been considered to be a potent tool for making an easy and quick evaluation of the growth fraction. The expression of Ki-67 varies in means of intensity throughout the different stages of cell cycle. Ki-67 antigen, which may be detected in all stages of cell cycle except G0, is associated with tumor proliferation, invasiveness, and ultimately prognosis (O.M. Wahba et al, 2013). So, Ki-67 has been used to determine the proliferation rate of many tumors and predict their biological behavior (A. Abdel Aziz and M.M. Amin, 2012).

Matrix metalloproteinases (MMPs), a group of zinc dependent neutral endopeptidases, have the ability to degrade ECM components, including collagens, elastin, proteoglycans, laminin, and fibronectin in addition to nonmatrix proteins as growth factors, cytokines, chemokines, and their receptors. Thus, they have a role in regulating cell growth and inflammation (S. De and J.E. Fenton, 2005).

Physiologically, MMPs have a key role in conditions depending on degradation of ECM like angiogenesis, tissue repair, and tissue morphogenesis (H. Nagase and J.F. Jr Woessner, 1999). Depending on their function, the gene family can be divided into the subgroups of collagenases, gelatinases, stromelysin, matrilysins, membranetype MMPs, and other MMPs (R. Visse and $\boldsymbol{H}$. Nagase, 2003).

Matrilysin-1 (MMP-7) is primarily expressed by glandular epithelial cells in the endometrium, small intestine, breast, pancreas, liver, prostate, dermis, bronchus, and by epithelial tumors of the gastroin- testinal tract, prostate, and breast (P. Vihinen et al, 2005; U.K. Saarialho-Kere et al, 1995).

MMP-7 has the ability to activate antibacterial peptides. So, it plays a role in intestinal mucosal defense (C.L. Wilson et al, 1999). Besides ECM components, MMP-7 is known to degrade cell surface molecules such as Fas-ligand, pro-tumor necrosis factor (TNF)- $\alpha$, and E-cadherin ( $\boldsymbol{R}$. Visse and $\boldsymbol{H}$. Nagase, 2003).

The purpose of this study is to correlate the clinical data of patients with MSGTs regarding lymph node metastasis with the expression of Ki-67 and MMP-7 proteins using immunohistochemistry.

\section{MATERIAL AND METHODS}

Ragents

1- Primary Antibodies: the antibodies used in this study were a mouse monoclonal antibody MMP-7 (Santa Cruz Biotechnology Inc., CA) and mouse monoclonal antibody Ki-67(clone MIB-1, IS626, Ready to use, Dako, Denmark).

2- Secondry antibody: Envision FlexL / HRP goat secondary antibody molecules (DM822, Dako, Denmark) were used.

3- Detection system: Dako Envision ${ }^{\mathrm{TM}}$ Flex detection system was used for immune-detection.

\section{METHODS}

\section{Specimens selection:}

Thirty formalin fixed and paraffin-embedded specimens of malignant salivary gland tumors were collected from the archives of Oral and Maxillofacial Pathology Department, Faculty of Dentistry, Minia University and Clinical Pathology Department of The National Institute of Cancer, Minia.

Clinical records for the patients were reviewed and information regarding site, sex, age, lymph node metastasis and clinical stage was collected. 
Each case was coded and patient's name was not shown for ethical reasons.

$4 \mu \mathrm{m}$ thick sections were cut and mounted on glass slides; sections were stained with haematoxylin and eosin stain and examined by light microscope. According to the histopathological criteria and based on WHO, slides were classified into different types of malignant salivary gland tumors.

\section{Immunohistochemistry:}

For all specimens, $4 \mu \mathrm{m}$ sections were cut and mounted on positively charged glass slides. The cutting sections were de-waxed in xylene, and rehydrated in graded alcohol.

Blocking of endogenous peroxidase activity was done by applying of $3 \%$ hydrogen peroxide for 5 minutes at room temperature, then the slides were washed by phosphate buffer solution. For antigen retrieval, we used Envision ${ }^{\mathrm{TM}}$ FLEX Target Retrieval solution, high pH (pH 9) (Dako, Denmark) with concentration 1:50 using distilled water for staining procedure. Using a heater, we preheated the solution to $65^{\circ} \mathrm{C}$ and then the slides were immersed and incubated for 20 minutes at $97^{\circ} \mathrm{C}$.

Two primary antibodies were used, flex monoclonal mouse anti-human ready to use Ki-67 antibody (Ready to use, Dako, Denmark) and monoclonal mouse anti-human MMP-7 primary antibody (Santa Cruz Biotechnology Inc., CA) with a dilution $1 / 100$.

The slides were incubated at $4^{\circ} \mathrm{C}$ overnight. Next day, the slides were incubated with secondary antibody (HRP) for 20 minutes. A 3-3 ,diaminobenzidine tetrahydrochloride hydrate substrate (Dako, Denmark) was used to visualize the reaction.

Finally, the sections were counterstained with Mayer's hematoxylin. All steps were performed according to manufacture instruction guide.

Proper positive and negative controls were used. Tonsil tissue and breast cancer tissue served as positive control for Ki-67 and MMP-7, respectively (according to manufacture recommendation), while negative controls were obtained by omission of the primary antibodies, which were substituted with $1 \%$ phosphate buffered saline (PBS).

\section{Evaluation}

For immunostaining analysis, the slides were examined with a light microscope (Leica, Germany). Tissue sections were scanned at X100 magnification for the highest immunopositive areas, five microscopic fields for each case were selected at a magnification of X400. Photomicrographs were captured using a digital camera mounted on the light microscope.

\section{Semiquantitative Evaluation of MMP-7 Staining.}

Images were then transferred to the computer system for analysis. Immunoreactivity of MMP-7 was evaluated using index of positivity (IP) obtained by multiplication of the intensity of cytoplasmic staining $(0=$ no staining, $1=$ mild, $2=$ moderate, and $3=$ strong cytoplasmic staining) by the percentage of positively stained cells.

\section{Evaluation of Ki-67 Staining}

Ki-67 immunoreactivity was evaluated using area fraction (percentage of immunopositive area to the total area of the microscope field). This was carried out using image analysis software (Image J, 1.41a, NIH, USA).

\section{Statistical Analysis}

The data was stored and analyzed and graphics were done by The Statistical Program SPSS for Windows version 22. Student t-test was used to compare between two means. Anova test was used for comparison of more than two means. Pearson correlation analysis was used to describe the association between numerical variables. The results were considered significant for $\mathrm{p} \leq 0.05$. 


\section{RESULTS}

The study subjects consisted of 30 blocks of 11 men and 19 women with mean age at time of diagnosis of 57 (range, 27-88 years). A total of 12 cases of malignant pleomorphic adenoma, 7 cases of adenoid cystic carcinoma (ACC) and 11 of mucoepidermoid carcinoma (MEC) were identified.

Of the 30 cases, 4 (13.33\%) had tumors categorized as clinical stage I, 10 (33.33\%) had stage II tumors, $9(30 \%)$ had stage III tumors, and 7 (23.34\%) had stage IV tumors. 16 cases showed lymph node metastasis (confirmed radiographically and pathologically).

Positive Ki-67 and MMP-7 expression was found in all cases examined in the present study. Positive cases showed homogenous and brownish immunostaining of tumor cells.

\section{Ki-67 immunostaining:}

The immunostaining was mainly nuclear (Fig.1). The highest staining intensity was observed with sections of adenoid cystic carcinoma (22.58 \pm 0.48$)$. The epidermoid cells of mucoepidermoid carcinoma showed Ki-67 staining while mucous cells were not stained (21.53 \pm 1.52$)$. Cases of malignant pleomorphic adenoma showed the least Ki-67 expres-

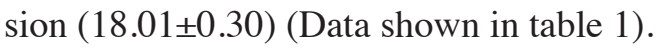

\section{MMP-7 immunostaining}

The staining pattern was, in general, cytoplasmic and varied from weak to strong (Fig. 2). The IP of MMP-7 ranged from 0 to 300. The strongest staining was observed with cases of adenoid cystic carcinoma (276.86 \pm 8.25$)$.

Cases of malignant pleomorphic adenoma showed MMP-7 expression in the epithelial and ductal cells (Fig. 2C) with a mean of IP of 207.25 \pm 8.91 . The epidermoid cells of MEC showed MMP-7 staining (Fig. 2B) with a mean of $253.18 \pm 12.62$. Mucous and clear cells were not stained. All cases of ACC exhibited moderate/severe MMP-7 expression (Fig. 2A) (Data shown in table 2).

\section{Statistical results:}

Pearson correlation test showed a positive correlation between Ki-67 and MMP-7 expression $(p$ value $=0.0001)($ fig. 3$)$.

The relationship between Ki-67 and MMp-7 expression and lymph node metastasis was statistically significant (table 1,2 ). No significant relation was found between $\mathrm{Ki}$ and MMP-7 expression and either sex or site of malignant salivary gland tumors. Conversely, the expression of both markers was related significantly to the type of the tumor (table 1,2).

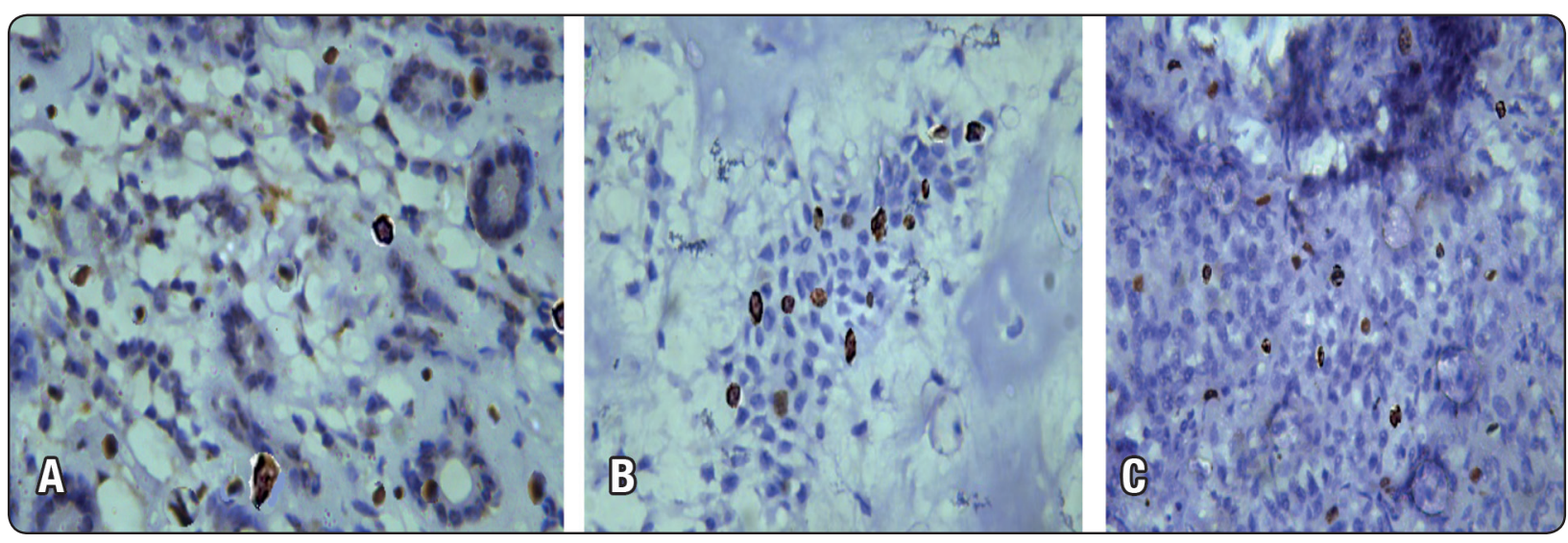

Fig. (1): Ki-67 expression in malignant salivary gland tumors. A. Adenoid cystic carcinoma. B. Mucoepidermoid carcinoma. C. Malignant Pleomorphic adenoma (Immunostaining, 400X) 


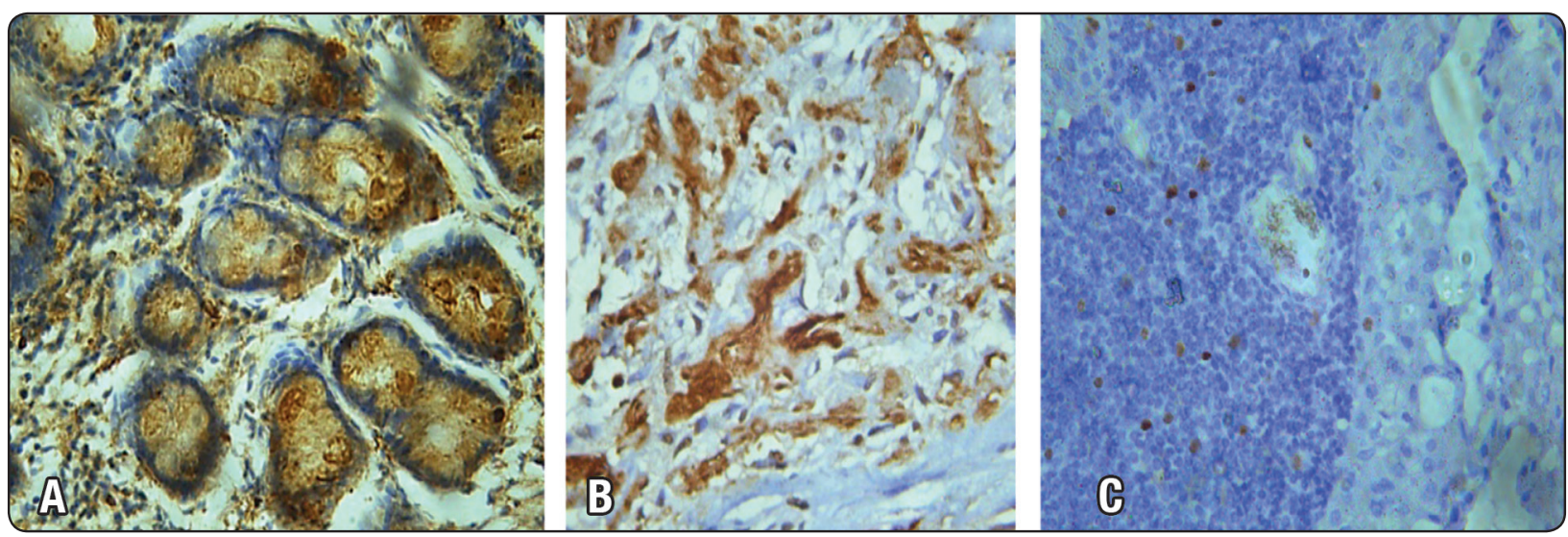

Fig . (2): MMP-7 immunostaining in malignant salivary gland tumors. A. Adenoid cystic carcinoma. B. Mucoepidermoid carcinoma. C. Malignant pleomorphic adenoma (Immunostaining, $400 \mathrm{X}$ ).

TABLE (1): Relation between Ki-67 expression and clinicopathological characters of MSGTs

\begin{tabular}{|l|c|c|c|}
\hline \multicolumn{1}{|c|}{ Variable } & No of patients & Staining intensity of Ki-67 & P value \\
\hline All cases & 30 & & \\
\hline Type & 12 & $18.01 \pm 0.30$ & \\
Malignant pleomorphic adenoma & 11 & $21.53 \pm 1.52$ & 0.0001 \\
Mucoepidermoid carcinoma & 7 & $22.58 \pm 0.48$ & \\
Adenoid cystic carcinoma & 11 & $20.78 \pm 1.96$ & 0.4 \\
\hline Sex & 19 & $20.13 \pm 2.48$ & 0.2 \\
Male & 18 & $19.97 \pm 2.45$ & \\
Female & 12 & $20.97 \pm 1.99$ & 0.0001 \\
\hline Site & 14 & & \\
Major salivary gland & 16 & $22.56 \pm 0.56$ & \\
Minor salivary gland & & $18.45 \pm 1.23$ & \\
\hline Lymph node metastasis & & & \\
Present & & & \\
Absent & &
\end{tabular}

TABLE (2): Relation between MMP-7 IP and clinicopathological characters of MSGTs

\begin{tabular}{|l|c|c|c|}
\hline Variable & No of patients & IP of MMP-7 & P value \\
\hline All cases & 30 & & \\
\hline Type & 12 & $207.25 \pm 8.91$ & \\
Malignant pleomorphic adenoma & 11 & $253.18 \pm 12.62$ & 0.0001 \\
Mucoepidermoid carcinoma & 7 & $276.86 \pm 8.25$ & \\
Adenoid cystic carcinoma & 11 & $243.91 \pm 27.96$ & 0.6 \\
\hline Sex & 19 & $238.26 \pm 32.59$ & \\
Male & 18 & $235.83 \pm 31.172$ & 0.3 \\
Female & 12 & $47.08 \pm 28.83$ & \\
\hline Site & 14 & $268.92 \pm 11.01$ & 0.0001 \\
Major salivary gland & 16 & $215.31 \pm 16.57$ & \\
Minor salivary gland & & & \\
\hline Lymph node metastasis & & & \\
Present & & & \\
Absent & & & \\
\hline
\end{tabular}




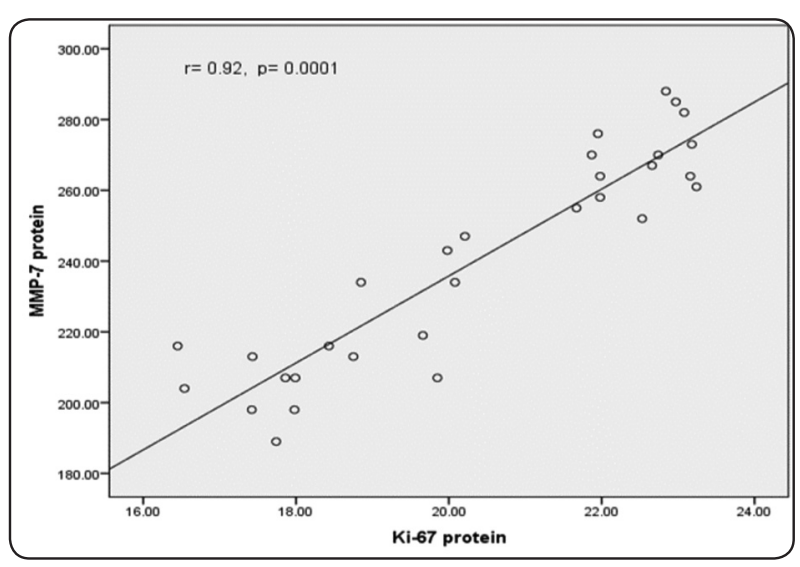

Fig. (3): Scatter plot diagram showing the correlation between Ki-67 and MMP-7 expression

\section{DISCUSSION}

MMP have prognostic value in different human malignancies including head and neck squamous cell carcinoma (P.O-Charoenrat et al, 2002).

The correlation of Ki-67 and MMP-7 expression with lymph node metastasis in malignant salivary gland tumors has not been studied before. In the present study of 30 cases with MSGTs, the staining pattern of both Ki-67 and MMP-7 was described and the results were linked to lymph node metastasis and some clinical parameters.

The index of positivity of MMp-7 was highest in adenoid cystic carcinoma followed by mucoepidemoid carcinoma and then malignant pleomorphic adenoma.

A statistically significant association was found between MMP-7 expression and lymph node metastasis. This discovery was consistent with the report of Shuo et al. (Y. Shuo et al, 2020) which showed that increased lymph node metastasis was associated with elevated expression of MMP-7 in tongue squamous cell carcinoma.

Barros et al found that enhanced expression of MMP-7 promoted cell proliferation, migration and invasion (S.S. Barros et al, 2011). Thus, it plays a role in tumor progression. This may explain the high MMP-7 IP in adenoid cystic carcinoma which is more aggressive than other studied malignant salivary gland tumors.

Other reports showed that serum levels of MMP7 were significantly higher in patients with bladder cancer with metastatic disease, lymphovascular involvement, and lymph node metastasis than in control groups (M. Gunes et al, 2013).

The mechanism of action of MMP-7 includes indirect destruction of the vital components of the extracellular matrix by activating other individual MMPs to promote tumor cell metastasis. MMP7 activates gelatinases (MMP-2 and MMP-9) and enhances MMP-1 activity. Furthermore, it partially activates pro-MMP-9 (Y. Shuo et al, 2020).

MMP-7 is correlated with tumor-induced neovascularization as the endothelial progenitor biomarker, CD34 was discovered in renal cell carcinoma expressing MMP-7 (Y. Miyata et al, 2006).

These results show that MMP-7 functions as an oncogene in malignant tumors and could be used as a therapeutic target.

No significant correlation was found between MMP-7 IP and either sex or site of the tumor. Similar results were found in a study which explored the correlation of MMP-7 in colorectal cancer with clinical parameters (Z. Bi et al, 2013)

Similarly, results observed with Ki-67 immunostaining were correlated with lymph node metastasis and type of the tumor but not affected with either sex or site of the tumor. Our results were in accordance with that study of $\mathrm{H}$. Luukkaa et al. who found that Ki-67 expression is correlated with prognosis in malignant salivary gland tumors $(\boldsymbol{H}$. Luukkaa et al, 2006).

Ki-67 expression was significantly related to MMP-7 expression in our studied cases. That finding is in accordance with that of L Dage et al. who concluded that MMP-7 overexpression was associated with tumor proliferation and poor prognosis in non-small lung cancer (L. Dage et al, 2007). 


\section{RECOMMENDATIONS}

The present study showed that MMP-7 may have a prognostic impact in malignant salivary gland tumors but the exact mechanism needs to be elucidated and this may require further studies.

\section{REFERENCES}

- A. Abdel-Aziz and M.M. Amin, 2012 "EGFR, CD10 and proliferation marker Ki67 expression in ameloblastoma: possible role in local recurrence". Diagnostic Pathology, 7(1), article 14.

- A. Rousseau and C. Badoual, 2011" Head and neck: salivary gland tumors: an overview". Atlas Genet Cytogenet Oncol Haematol.;15(6):534e541.

- A.L.D. Kruse, K.W. Grätz, J. A. Obwegeser and H.T. Lübbers, 2010 "Malignant minor salivary gland tumors: a retrospective study of 27 cases" , Oral Maxillofac Surg, 14:203-209.

- C.L. Wilson, A.J. Ouellette and D.P. Satchell, 1999 "Regulation of intestinal alpha-defensin activation by the metalloproteinase matrilysin in innate host defense". Science; 286:113-117.

- H. Luukkaa, P. Klemi, I. Leivo, T. Vahlberg and R. Grénman, 2006 "Prognostic significance of Ki-67 and p53 as tumor markers in salivary gland malignancies in Finland: an evaluation of 212 cases". Acta Oncol (Stockholm, Sweden);45:669-675.

- H. Nagase and JF Jr Woessner, 1999 "Matrix metalloproteinases”. J Biol Chem; 274:21491-21494.

- L. Dage, N. Jun, I. Sinya, Y. Hiroyasu, U. Masaki, K. Kyuichi, U. Masahide and H. Cheng-long, 2007 “Overexpression of matrix metalloproteinase-7 (MMP-7) correlates with tumor proliferation, and a poor prognosis in nonsmall cell lung cancer". Journal of Lung Cancer, Volume 58, Issue 3, Pages 384-391.

- M. Gunes, A.S. Kemik, N. Pirincci, I. Gecit, K. Taken, M.B. Yuksel, M. Kaba and R. Eryilmaz, 2013 "Preoperative levels of matrix Metalloproteinase-7 and -9 and tissue inhibitor of matrix Metalloproteinase-1 relation to pathologic parameters in bladder carcinoma patients". Asian Pac J Cancer Prev.;14(2):873-6.

- O.M. Wahba, A.M. Raghib, E.M. Megahed and M.M. Hus- sein, 2013 “Expression of perlecan, syndecan-1 and Ki-67 in keratocysticodontogenic tumor". Tanta Dental Journal, 10(3).153-159.

- $\quad$ P. O-Charoenrat, P. Rhys-Evans and S. Eccles, 2002 “A synthetic matrix metalloproteinase inhibitor prevents squamous carcinoma cell proliferation by interfering with epidermal growth factor receptor autocrine loops". Int J Cancer; 100:527-533.

- $\quad$ P. Vihinen, R. Ala-aho and V.M. Ka“ha" ri, 2005 "Matrix metalloproteinases as therapeutic targets in cancer". Curr Cancer Drug Targets; 5:203-220.

- P.C.Anila Namboodiripad, 2014 “ A review: Immunological markers for malignant salivary gland tumors". Journal of oral biology and craniofacial research, 4: $127-134$.

- $\quad$ R. Visse and H. Nagase, 2003 "Matrix metalloproteinases and tissue inhibitors of metalloproteinases: structure, function, and biochemistry". Circ Res; 92:827-839.

- $\quad$ S. De, J.E. Fenton and A.S. Jones, 2005 “ Matrix metalloproteinases and their inhibitors in non-neoplastic otorhinolaryngological disease”. J Laryngol Otol 119:436-442.

- S.S. Barros, A.C. Henriques, K.M. Pereira, A.M. de Medeiros, H.C. Galvao and A. Freitas Rde, 2011 "Immunohistochemical expression of matrix metalloproteinases in squamous cell carcinoma of the tongue and lower lip". Arch Oral Biol.; 56(8):752-60.

- U.K. Saarialho-Kere, E.C. Crouch and W.C. Parks, 1995 "Matrix metalloproteinase matrilysin is constitutively expressed in adult human exocrine epithelium". J Invest Dermatol; 105:190-196.

- $\quad$ Y. Miyata, T. Iwata, K. Ohba, S. Kanda, M. Nishikido and H. Kanetake, 2006 "Expression of matrix metalloproteinase-7 on cancer cells and tissue endothelial cells in renal cell carcinoma: prognostic implications and clinical significance for invasion and metastasis". Clin Cancer Res.;12(23):6998-7003.

- Y. Shuo, L. Li-song, G. Rui-Huan, H. Li, W. Xiao-ting, Z. Yong, S. Bo-hua, Z. Dali and L. You-Guang, 2020 "Elevated matrix metalloproteinase 7 expression promotes the proliferation, motility and metastasis of tongue squamous cell carcinoma”. BMC Cancer, 20:33.

- Z. Bi, L.D. Dong and X.M. Gu, 2013 "Clinical significance of MMP-7 and PTEN expression in colorectal cancer." Hepatogastroenterology. Jan-Feb;60(121):32-6. 